\title{
Carnets
}

Revue électronique d'études françaises de l'APEF

Deuxième série - 8 | 2016

Du Français en cause aux causes du français

\section{Approches politiques de la situation du français dans le monde}

Yves Montenay

\section{(2) OpenEdition}

Journals

Édition électronique

URL : http://journals.openedition.org/carnets/1956

DOI : $10.4000 /$ carnets. 1956

ISSN : 1646-7698

\section{Éditeur}

APEF

Référence électronique

Yves Montenay, «Approches politiques de la situation du français dans le monde », Carnets [En ligne], Deuxième série - 8 | 2016, mis en ligne le 30 novembre 2016, consulté le 23 avril 2019. URL : http:// journals.openedition.org/carnets/1956; DOI : 10.4000/carnets.1956

Ce document a été généré automatiquement le 23 avril 2019.

\section{(c) (i) (8)}

Carnets est mis à disposition selon les termes de la licence Creative Commons - Atribution - Pas d'utilisation commerciale 4.0 International. 


\title{
Approches politiques de la situation du français dans le monde
}

\author{
Yves Montenay
}

1 Cet article est une présentation partielle de mon livre «La langue française, arme d'équilibre dans la mondialisation ", que Les Belles-Lettres distribueront à partir février 2015. Je vais me limiter à quelques grands traits de la situation mondiale du français et des questions politiques qui y sont liées.

2 Cette situation est mal connue, et je vais essayer de la "recadrer » historiquement et géopolitiquement.

\section{Des handicaps dus à des causes extérieures, mais également intérieures}

\section{Les causes extérieures}

3 On oublie souvent que des populations francophones ont été particulièrement visées par des violences $\mathrm{du} \mathrm{Xx}^{\mathrm{e}}$ siècle, pendant lequel elles se sont fait massacrer ou disperser.

4 La première guerre mondiale a fait disparaître une grande partie de l'élite européenne qui était francophone. On sait que les officiers étaient souvent en tête de leurs troupes et ont été particulièrement fauchés dans les premières semaines, alors qu'on avait mal évalué la puissance de feu du matériel moderne. En France, les publications des élèves des grandes écoles font allusion à des promotions presque complètement massacrées; en Autriche j'ai noté dans les mémoires des passages relatifs à ses camarades de la bonne bourgeoisie viennoise « destinés à être les grands cadres de la nation, mais qui ont tous étés tués ».

5 Les révolutions, la deuxième guerre mondiale et les guerres civiles s'y sont ajoutées, avec l'élimination physique ou l'exil des noblesses et bourgeoisies francophones : élimination quasi totale en Russie de 1917 à la fin de la guerre civile, attentats ciblés puis épuration politique au Vietnam, au Cambodge (dans les camps khmers rouges: "que ceux qui 
savent parler français lèvent la main, ils auront un travail intéressant » et ceux qui s'étaient ainsi désignés étaient exécutés). Départ pour l'exil, au Laos, en Égypte et Iran, et dans une large mesure au Liban, au Maghreb, en Guinée, à Madagascar et en Europe centrale et orientale : les francophones étaient soient «un colon » (même s'il s'agissait d'un employé de l'institut Pasteur), soit « un féodal » ou « un bourgeois » (comprendre un ennemi de classe à éliminer, ou tout simplement, un appartement à récupérer en menaçant son propriétaire), soit encore un intellectuel potentiellement hostile au nouveau gouvernement. Précisons que tout cela a eu lieu non seulement pendant les révolutions ou guerres d'indépendance, mais également plus longtemps après lors de divers soubresauts politiques.

6 Tout cela sans compter l'action «normale» des langues concurrentes et des États les soutenant, notamment à Bruxelles, en Afrique et dans bien d'autres pays du Sud. En Iran, par exemple, avant la révolution islamiste qui a éliminé beaucoup de cadres, francophones comme anglophones, l'arrivée des institutions américaines à partir de 1947 (élimination par la CIA du dirigeant francophone Mossadeg) s'est traduite par un déluge de bourses et d'actions culturelles qui ont dirigé vers l'anglais une grande partie des étudiants iraniens, alors qu'auparavant, comme en Égypte, l'influence britannique n'avait pas gagné le domaine linguistique et les couches moyennes et supérieures étaient donc francophones. Malgré les tensions aiguës entre la république islamique et les États-Unis, l'action des universités et fondations américaines s'est poursuivie, et les meilleurs étudiants se voient proposer des bourses pour les bonnes universités américaines.

\section{Les causes intérieures}

7 La plus importante est l'évolution démographique : la France est passée de $20 \%$ à $1 \%$ de la population des pays avec lesquels elle était en contact commercial ou guerrier, l'Europe hier, le monde entier aujourd'hui.

8 Et, ce qui est moins connu en dehors des cercles économiques, l'état d'esprit de l'intelligentsia française. Le colbertisme puis le jacobinisme, suivi du blocus napoléonien a freiné un premier développement économique, et donc le poids et l'influence du pays. Après une éclaircie, notamment sous Louis-Philippe, Napoléon III et le début de la III ${ }^{e}$ République qui ont vu l'économie française rattraper une partie de son retard lors de cette première mondialisation, avec comme symbole Gustave Eiffel, dont la tour n'est qu'une illustration publicitaire de sa percée dans l'industrialisation de la construction métallique (des ponts dans le monde entier, des plus modestes aux plus gigantesques) puis dans les souffleries de conception des avions. La disparition d'une partie des élites pendant la première guerre mondiale a donné un coup d'arrêt à ce développement économique, mais le phénomène a été aggravé par l'arrivée au pouvoir politique ou intellectuel de générations communistes et socialistes. Sans vouloir faire ici de politique, je me borne à constater que cela a poussé la classe entrepreneuriale à estimer que le système économique des pays anglophones était meilleur et à en adopter les techniques "de management» et la langue. Les maladresses du gouvernement actuel sont de nouvelles illustrations de cette vision du monde, qui vide la France au bénéfice notamment de Londres.

9 Précisons que les pays anglophones en question sont d'abord les États-Unis qui ont profité des deux guerres mondiales, et que la Grande-Bretagne a dans un premier temps subi le même déclin relatif que la France et pour les mêmes raisons, avant son redressement sous 
les gouvernements successifs de Thatcher, Blair et Cameron. Toujours en restant au niveau des faits économiques et en évitant la politique pure, j'ai constaté qu'avant ces gouvernements les Anglais cherchaient du travail dans le monde entier, en France, mais aussi dans la péninsule arabique où ils avaient un statut intermédiaire entre «les expatriés » français ou américains et les " esclaves » pakistanais, et que, maintenant, ce sont les Français qui vont chercher du travail en Grande-Bretagne.

\section{Les éléments positifs}

- la plus importante a été "la grande coopération» au Maghreb et en Afrique subsaharienne autour des années 1960 et 70. Des dizaines de milliers de Français ont formé les cadres du Maghreb et de l'Afrique subsaharienne, et ont assisté des nouveaux responsables politiques et administratifs. Les élèves ainsi formés ont été les cadres des 40 dernières années et ont souvent transmis leur connaissance du français à leurs employés. Néanmoins, des coopérants prennent maintenant leur retraite et leurs successeurs ont souvent été moins bien scolarisés, sauf la minorité passée par l'enseignement privé ... Et qui n'a pas émigré pour les raisons signalées ci-dessus.

- le lancement de RFI, de TV5 et de France 24

- la coopération décentralisée, qui met en contact professionnel des cadres moyens et les techniciens des municipalités françaises et locales

Mais tout cela ne constitue pas une politique, notamment par manque de conviction profonde. En particulier il est dommage de voir le terme « francophonie » monopolisé de fait par l'OIF. Cela d'abord en termes d'image : une organisation, ce sont des bureaux, ça ne frappe pas l'imagination; évoquer «les peuples francophones " auraient été plus parlant. Mais également, au-delà de l'image, parce que le gros des problèmes est hors de la portée d'une simple organisation. En effet si la démographie africaine génère des centaines de millions de francophones potentiels, elle complique énormément la concrétisation de ce même potentiel et le problème dépasse maintenant aussi bien l'OIF que la France.

\section{La démographie africaine est à double tranchant}

12 L'attention se porte donc trop sur ce que devrait faire la France, alors que l'essentiel est maintenant ailleurs : si l'Afrique "francophone" parvient à le rester, et a le poids démographique que l'on prévoit (750 millions vers 2050), toute action française (ou québécoise, ou belge...) ne sera pas à l'échelle du problème : pensez à la formation de centaines de milliers d'instituteurs et d'enseignants du secondaire, à celle de dizaines de milliers de fonctionnaires municipaux. Les Portugais, avec leur faiblesse démographique et économique actuelle et leur lusophonie brésilienne et africaine très supérieure à la population du Portugal, me comprendront.

13 L'action de la France ne pourrait être dans le meilleur des cas qu'incitative ou politicopsychologique, comme l'opération Serval (et sa suite) au Mali en donne un bon exemple. Rappelons que l'armée française alliée à celle d'autres pays francophones, est intervenue à la demande du gouvernement malien et de l'ONU pour mettre fin à des " mouvements » où se combinaient djihadistes, rivalités ethniques et trafics d'êtres humains, de drogue et

Carnets, Deuxième série - 8 | 2016 
d'armes, "mouvements» qui après avoir pris le contrôle du nord de ce pays se dirigeaient vers sa capitale. Au-delà de cet objectif, avoir pu compter sur la France et la coopération entre francophones semble avoir été bénéfique au statut du français même si ce n'était pas son objectif principal. Accessoirement, j'espère que les Américains ont remarqué que coopérer avec une population dont les cadres parlent la même langue que l'armée envoyée facilite les choses.

Mais il y a encore des obstacles psychologiques au Nord comme au Sud à cette prise de conscience d'unité des francophones. Au Nord se développe un courant isolationniste face à la pression migratoire du Sud, tandis qu'au Sud on évoque l'illégitimité de la «langue coloniale » question depuis longtemps dépassée puisqu'elle a été adoptée par les élites et le peuple depuis bientôt 60 ans. Par ailleurs, plus le temps passe et plus un éventuel changement de langue pénaliserait les cadres du pays, et son développement. Mais comment être certain de la rationalité de tous les futurs dirigeants africains?

L'avenir du français en tant que langue de masse à l'échelle mondiale sera donc ce qu'en feront les pays africains francophones. Ils doivent se prendre en main sans compter sur des actions françaises, qui ne pourront pas faire de miracle. Les plus solides devraient même coopérer culturellement et économiquement avec les autres. La politique du Maroc dans ce domaine est un bon exemple, avec l'action de Mohamed VI sur le plan politicoreligieux et en appui des entreprises marocaines qui s'implantent au sud du Sahara, et qui sont francophones de fonctionnement.

Pour l'instant, le complexe « formation, entreprises et médias » fonctionne efficacement : la formation professionnelle privée produit des francophones parce que c'est ce qui permet l'emploi, et les entreprises francophones se développent parce que cette formation professionnelle existe, tandis que les médias visant ces classes entretiennent le tout en français. Mais ces trois acteurs sont principalement privés et suivront peut-être la mode de l'anglicisation, tout comme certains acteurs publics. Le mépris de l'économie et des affaires par une partie de l'élite française est un mauvais exemple.

\section{Et la francophonie hors d'Afrique?}

17 Les masses en jeu étant moins importantes, on est ramené à des actions plus classiques de la France, du Québec, de l'OIF etc. Mais un des facteurs de diffusion du français, son rôle économique, qui dépendra en partie du nombre de francophones africains. On me dit que les Portugais trouvent des emplois au Maghreb grâce au français. Et si 200000 (?) étudiants et des cadres chinois l'apprennent, c'est aussi largement pour travailler en Afrique.

\section{Conclusion}

Le français langue de culture a été victime de l'élimination des classes moyennes supérieures de beaucoup de pays. Souhaitons qu'il n'en aille pas de même en Afrique où il est bien installé dans ces mêmes classes, mais où beaucoup de pays sont instables, tandis que des gouvernements sont sensibles à certains groupes d'intérêts (le Ruanda, mais aussi la RDC et Madagascar où cela a été rattrapé). Par contre, la réussite africaine donnerait un poids mondial au français économique comme culturel. 
Quant à la France, ses gouvernements manquent de volonté politique dans ce domaine depuis quelques décennies. S'y ajoutent de la part du gouvernement actuel des erreurs économiques qui affaiblissent aussi la langue, et confirment dans la classe managériale l'impression que le système anglophone est plus efficace. Cela à tort (voir mes travaux) comme certains commencent à s'en rendre compte.

La France s'est redressée plusieurs fois dans le passé, et le présent n'implique pas l'avenir. Reste à informer les gouvernements futurs de l'importance de ces questions.

\section{RÉSUMÉS}

Cet article est une présentation partielle de mon livre La langue française, arme d'équilibre de la mondialisation, Les Belles-Lettres 2015. Elle rappelle les événements historiques "extérieurs » (guerres, révolutions...) et les données intérieures (démographie, politique économique, coopération...) qui ont ébranlé ou renforcé la francophonie. Puis elle analyse la situation africaine, dont l'évolution aura un poids déterminant, et le rôle que pourrait y jouer la France et les pays du Nord: cette masse de francophones potentiels dépasse maintenant beaucoup d'instruments traditionnels et attire l'attention d'acteurs étrangers, parfois adversaires de la francophonie.

Nous mettrons l'accent sur les données économiques, notamment sur l'état d'esprit des entreprises et leur rôle important, tant positif que négatif pour l'enracinement du français.

This paper presents the main points of La langue française, arme d'équilibre de la mondialisation (The French language, a weapon to balance globalization), Les Belles-Lettres, Paris, 2015. This book examines the impact of extrinsic events (wars, revolutions...) as well as intrinsic systems (demography, economics, North-South cooperation...) on the worldwide use of French. It then analyses how this language is rooted in Africa and the crucial importance of the coming years in African countries for French's worldwide destiny. But helping the training in French of those hundreds of millions of (more or less) French-speaking people, exceeds what the traditional cooperation with France and other northern francophone countries is able to do, while, simultaneously, those African countries attract the interests of foreign economic actors, even those opposed to the spread of French.

The paper will also point up how companies and private business does root the use of French, but sometimes also spread the use of English.

\section{INDEX}

Keywords : Africa, francophonie, cooperation, globalization, working language

Mots-clés : Afrique, francophonie, coopération, mondialisation, langue de travail 


\section{AUTEUR}

\section{YVES MONTENAY}

Institut Culture, Économie et Géopolitique (Paris)

montenay[at]numericable.fr 